\title{
Dynamic networks reveal key players in aging
}

\author{
Faisal, F. E. and Milenković, T.* \\ Computer Science and Engineering, University of Notre Dame, Notre Dame, IN 46556, USA
}

\begin{abstract}
Motivation: Since susceptibility to diseases increases with age, studying aging gains importance. Analyses of gene expression or sequence data, which have been indispensable for investigating aging, have been limited to studying genes and their protein products in isolation, ignoring their connectivities. However, proteins function by interacting with other proteins, and this is exactly what biological networks (BNs) model. Thus, analyzing the proteins' BN topologies could contribute to understanding of aging. Current methods for analyzing systems-level BNs deal with their static representations, even though cells are dynamic. For this reason, and because different data types can give complementary biological insights, we integrate current static BNs with aging-related gene expression data to construct dynamic, age-specific BNs. Then, we apply sensitive measures of topology to the dynamic BNs to study cellular changes with age.
\end{abstract}

Results: While global BN topologies do not significantly change with age, local topologies of a number of genes do. We predict such genes as aging-related. We demonstrate credibility of our predictions by: 1) observing significant overlap between our predicted aging-related genes and "ground truth" aging-related genes; 2) showing that our aging-related predictions group by functions and diseases that are different than functions and diseases of genes that are not predicted as aging-related; 3) observing significant overlap between functions and diseases that are enriched in our aging-related predictions and those that are enriched in "ground truth" aging-related data; 4) providing evidence that diseases which are enriched in our agingrelated predictions are linked to human aging; and 5) validating all of our high-scoring novel predictions via manual literature search.

Contact: tmilenko@nd.edu

\section{INTRODUCTION}

\subsection{Motivation and background}

Since the US is on average growing older because of $\sim 78$ million of baby boomers who have began turning 65 in 2011, and since susceptibility to diseases increases with age, studying human aging gains importance. Analysis of gene expression data has been indispensable for investigating aging (Wieser et al., 2011; Fortney et al., 2010). However, it has mostly been limited to studying differential expression of individual genes, without considering their connectivities (Fortney et al., 2010). But, it is the proteins (gene products) that carry out cellular processes and they do so by interacting with other proteins instead of acting alone. And this is exactly what biological networks and protein-protein interaction (PPI) networks in particular model; in PPI networks, nodes are proteins and edges correspond to physical interactions between the proteins. Thus, analyzing topologies of proteins in

\footnotetext{
*To whom correspondence should be addressed
}

PPI networks could contribute to understanding of the processes of aging. Although as a proof of concept this study focuses on PPI networks, it is applicable to other types of biological networks. High-throughput screens for PPI detection have yielded systemslevel (though incomplete) PPI networks for many organisms, which are publicly available (Breitkreutz et al., 2008).

The majority of current methods for analyzing systems-level PPI networks deal with their static representations, due to limitations of biotechnologies for PPI data collection, even though cells are dynamic (Przytycka and Kim, 2010). For this reason, and because different data types can give complementary biological insights (Memišević et al., 2010; Przytycka and Kim, 2010), we integrate current static PPI network data (Peri et al., 2004; Breitkreutz et al., 2008) with age-specific gene expression data (Lu et al., 2004) to computationally construct dynamic, age-specific PPI networks, in order to study cellular changes with age from such networks.

Furthermore, topological positions of aging-related genes in the static networks have been studied (Kriete et al., 2011; Ferrarini et al., 2005; Reja et al., 2009; Promislow, 2004; de Magalhães, 2009), but mostly with crude measures of topology that can not cope with the complexity of PPI networks (Pržulj, 2011). For example, node degrees have been used to argue the central role or aging-related proteins in the yeast network compared to proteins that are not associated with aging, or to study the role of chaperones (heat stock proteins) in aging (Promislow, 2004; Sőti and Csermely, 2007). In addition to aging, many approaches have aimed to link node degrees with, for example, essentiality (Jeong et al., 2001), disease (Sharan and Ideker, 2008; Vanunu et al., 2010), cancer (Jonsson and Bates, 2006; Aragues et al., 2008), or pathogenicity (Dyer et al., 2008). However, it is possible that the high-degree proteins have been more studied simply because of their known relevance to human health (Pržulj, 2011; Ratmann et al., 2009). Hence, more constraining measures of topology might be needed that go beyond capturing only the direct network neighborhood of a node (Milenković and Pržulj, 2008; Milenković et al., 2010a). While such measures exist and have been used to link proteins' network positions with their involvement in some biological processes (Sharan et al., 2007; Milenković et al., 2011), to our knowledge, they have not been linked to proteins' involvement in aging even in static and especially in dynamic PPI networks. Here, we apply a series of measures of topology, including some highly sensitive measures (Milenković and Pržulj, 2008; Milenković et al., 2011), to the dynamic PPI networks to identify key players in aging.

\subsection{Our study}

We aim to study human aging via integration of aging-related gene expression data with static PPI network data (Fig. 1). We obtain dynamic, age-specific PPI networks by selecting in the static network: 1) all proteins that correspond to actively expressed genes 


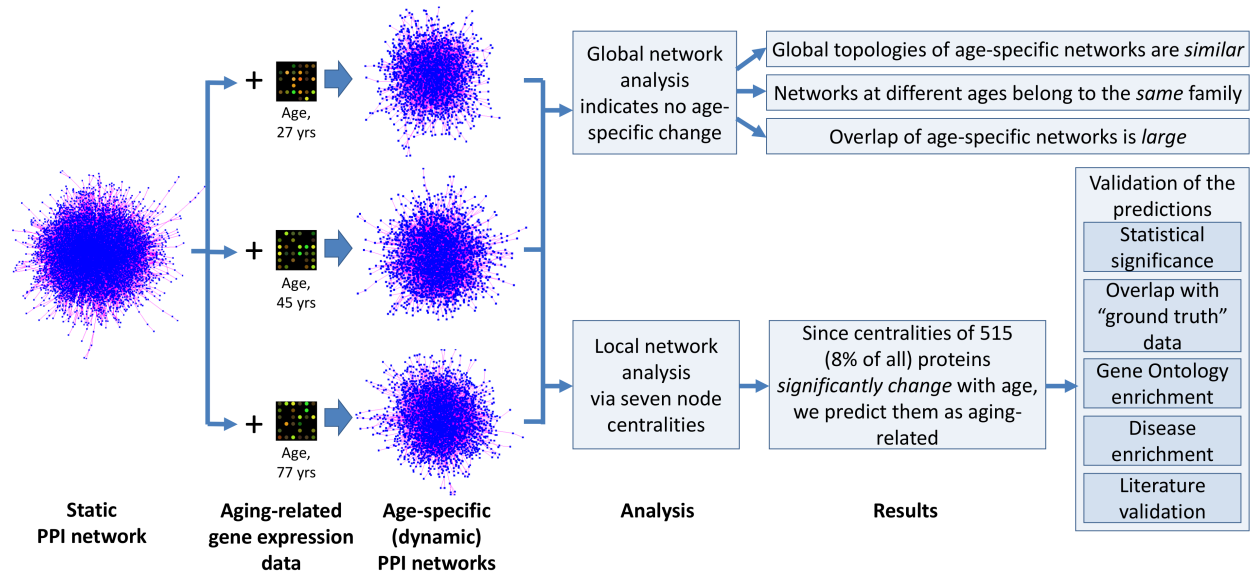

Fig. 1. Summary of our study. We integrate a static PPI network with aging-related gene expression data to obtain age-specific networks. We analyze changes in global and local network topologies with age. While global network analysis indicates no age-specific change, local topologies (as captured by seven node centrality measures) of some proteins do significantly change with age. We predict such proteins as agingrelated and validate our predictions in several ways.

at different ages and 2) all PPIs involving these "active" proteins. Hence, each age-specific network is the network that is "active" at a given age. We hypothesize that the dynamic and integrative network analysis provides a valuable model of cellular functioning that can reveal aging-related information and that can reveal more of the information than static analysis of individual data types.

Given the dynamic network data, we first aim to answer whether the overall network topologies change with age (Fig. 1). Since this is not the case, and since the gene expression data alone revealed only a small portion of all genes as aging-related (Lu et al., 2004), it could be that local topologies around only a subset of proteins in the network do change with age. Hence, we study positions of proteins in each age-specific PPI network with respect to measures of local topology, called node centralities, with the goal of identifying proteins whose centralities significantly change with age. We find 515 such proteins ( $8.1 \%$ of all proteins in the static network), which is quantitatively consistent to the result by $\mathrm{Lu}$ et al. (2004). We predict these proteins as aging-related and validate them as follows. 1) The predictions are statistically significant, i.e., non-random.

2) The overlap of our predicted aging-related genes and "ground truth" aging-related genes is significant. All of our five highestscoring predictions, namely GORASP2, MAP2K4, TIAM1, MAP1B, and S100B, are present in multiple aging-related "ground truth" data sets. Nonetheless, many of our predictions are novel, i.e., absent from the "ground truth" data. This confirms that dynamic network analysis of integrated data types can reveal additional biological knowledge compared to static analysis of individual data. 3) Our predictions group by biological functions and diseases that are different than functions and diseases of genes that we do not predict as aging-related. The overlap between functions and diseases that are enriched in our predictions and those that are enriched in the "ground truth" aging-related data is significant. Diseases that are enriched in our predictions are linked to human aging.

4) We manually search in the literature for our top $10 \%$ highestscoring predictions that are not present in the "ground truth" agingrelated data, and we successfully validate all of them.

\section{MATERIALS AND METHODS}

\subsection{Data}

2.1.1 Aging-related gene expression data We use human brain gene expression data consisting of 30 samples obtained from 30 individuals between 26 and 106 years of age. In an individual, Lu et al. (2004) defined a gene as being expressed (or active) at a given age if its detection p-value, which indicates the significance level of its mRNA abundance at that age, is less than 0.04. We adopt the same procedure (Supplementary Section S1.1).

2.1.2 Static PPI network data We obtain human static PPIs from HPRD (Peri et al., 2004) and BioGRID (Breitkreutz et al., 2008). HPRD data consists of 9,322 unique proteins and 36,030 unique PPIs between the proteins. BioGRID data consists of 10,078 unique proteins (with respect to their gene IDs) and 50,954 unique PPIs between the proteins.

2.1.3 Integrating static PPI network with gene expression data to form age-specific PPI networks We form dynamic, age-specific networks as follows. To form the network specific to a given age, we select in the static network those proteins that are expressed at that age (Section 2.1.1) and all PPIs that exist between the expressed proteins (see Supplementary Section S1.2 for a formal description). Since gene expression data is collected for 30 ages, 30 age-specific networks can be formed from the given static network. Since we study two static networks (HPRD and BioGRID), we obtain two sets of dynamic networks. We run subsequent analyses on each of the network sets. Since we find that results are similar across the two sets, for simplicity, here we report results only for the HPRD network. Results for the BioGRID network are reported in the Supplement.

2.1.4 "Ground truth" aging-related data We denote the set of genes present in both the static PPI network (Section 2.1.2) and brain gene expression data (Section 2.1.1) as StatNetExpression.

By studying brain gene expression data from Section 2.1.1, Lu et al. (2004) predicted 442 genes as aging-related, as their expression significantly correlated with age. Of these, 341 genes are present in StatNetExpression. Henceforth, we denote this "ground truth" aging-related set of 341 genes predicted from brain gene expression data alone as BrainExpression2004Age.

By studying another brain gene expression data with 174 samples from 55 individuals, with multiple sample per individual, Berchtold et al. (2008) identified 8,277 genes (via 12,514 probes) whose expression significantly changed with age. Of these, 3,228 are present in StatNetExpression. Henceforth, we denote this "ground truth" aging-related set of 3,228 genes predicted from brain gene expression data alone as BrainExpression2008Age.

Clearly, BrainExpression2004Age and BrainExpression2008Age are very similar in the sense that their aging-related genes have been inferred from brain gene expression data. (And as such, among all "ground truth" data sets (see below), these two sets are expected to be the most similar to our aging-related predictions, since our predictions are also partly based on brain human gene expression data.) However, it is important to note that BrainExpression2004Age and BrainExpression2008Age were predicted from two independent data sets, and compared to BrainExpression2004Age, BrainExpression2008Age is a result of a newer microarray study, it covers 
more samples and more individuals, and it covers more samples per individual.

By studying brain gene expression data set related to different stages of Alzheimer's disease (AD), Simpson et al. (2011) identified 2,911 genes (linked to 3,404 probes) that have significantly different expression levels at different stages of AD. Of these, 1,117 are present in StatNetExpression. Henceforth, we denote this "ground truth" AD-related set of 1,117 genes predicted from brain gene expression data as ADExpressionAge.

By studying gene expression data related to Hutchinson-Gilfold progeria syndrome (HGPS), a human premature aging-related disease, Liu et al. (2011) identified 1,731 genes that have a differentially methylated region between wild-type and HGPS-affected fibroblasts of vascular muscles cells. Of these, 708 are present in StatNetExpression. Henceforth, we denote this "ground truth" HGPS-related set of 708 genes predicted from vascular muscles-related gene expression data as HGPSExpressionAge.

In July 2012, GenAge contained 261 human genes that have been linked to aging as sequence-based orthologs of aging-related genes in model species (de Magalhães et al., 2009). Of these, 230 are present in StatNetExpression. Henceforth, we denote this "ground truth" aging-related set of 230 genes predicted from sequence data by SequenceAge.

2.1.5 Complements of the "ground truth" aging-related data We define a set of genes as the complement of a "ground truth" aging-related data set if the genes are present in StatNetExpression but not in the "ground truth" data set. We denote the complements of BrainExpression2004Age, BrainExpression2008Age, ADExpressionAge, HGPSExpressionAge, and SequenceAge as BrainExpression2004Complement, BrainExpression2008Con ADExpressionComplement, HGPSExpressionComplement, and SequenceComple respectively.

All above data sets are defined with respect to HPRD PPI data. For BioGRID data, see Supplementary Section S1.3.

\subsection{Do global network topologies change with age?}

Given the dynamic, age-specific PPI networks, we test whether the overall (global) topologies of the networks change with age. We do so by comparing the different networks with respect to several commonly used global network properties (Section 2.2.1), by evaluating the fit of each of the age-specific networks to a series of well-known graph families, i.e., network models (Section 2.2.2) (Milenković et al., 2008; Kuchaiev et al., 2011), and by measuring the overlap of the age-specific networks (Section 2.2.3).

2.2.1 Comparing global properties of age-specific networks We analyze three properties: the average clustering coefficient, average diameter, and graphlet frequency distribution (Memisević et al., 2010). The properties are defined in Supplementary Section S1.4.

2.2.2 Evaluating the fit of age-specific networks to different graph families or network models We compare the fit of the dynamic PPI networks to different graph families, i.e., network models (Milenković et al., 2009), to test whether the best fitting model changes with age. Various network models have been proposed. We use: (1) Erdös-Rényi random graphs (ER), (2) generalized Erdös-Rényi random graphs with same degree distribution as the data (ERDD), (3) geometric random graphs (GEO), (4) geometric gene duplication and mutation model (GEOGD), (5) scale-free networks (SF), and (6) scale-free gene duplication and mutation model (SFGD) (Milenković et al., 2008; Kuchaiev et al., 2011). To evaluate the fit of the data network to a given model, we compare the topology of the data network to the topology of a random network instance drawn from the model with respect to a highly constraining measure of network topological similarity called graphlet degree distribution agreement (GDD-agreement) (Pržulj, 2007). For details, see Supplementary Section S1.5.

2.2.3 Computing the overlap between age-specific networks We measure the overlap between each pair of age-specific networks as the percentage of nodes (or edges) in the smaller of the two networks that are common to the two networks. For details, see Supplementary Section S1.6.

\subsection{Do local topologies of proteins change with age?}

We study topological positions of proteins in each age-specific network with respect to seven node centrality measures (Section 2.3.1). We predict as aging-related those proteins whose centralities significantly change with age (Section 2.3.2). We validate our predictions in several ways (Section 2.3.3).

2.3.1 Local measures of topology or node centralities Various centrality measures have been used to link topological importance of a node in the network to its functional importance. Below, we define each of the seven measures that we use and provide biological justification for their use.

Degree centrality (DEGC) measures the degree of a node in the network, i.e., the number of the node's neighbors. The higher the degree of a node, the more central the node according to DEGC. Since current PPI networks have "power-law" degree distributions, with many low-degree nodes and few high-degree nodes, and since removal of the high-degree nodes would impact the network structure (by disconnecting it), DEGC of a gene has been related to the gene's essentiality as well as its involvement in disease (Barabási and Oltvai, 2004; Sharan and Ideker, 2008).

Clustering coefficient centrality (CLUSC) measures, for a given node, how many pairs of neighbors of the node are connected by an edge, out of all pairs of the node's neighbors. Intuitively, the more interconnected the neighborhood of the node, the more central the node is according to CLUSC. In a PPI network, a node with high clustering coefficient, together with the node's neighbors, forms a highly interconnected network region, which is likely to correspond to a functional module (Barabási and Oltvai, 2004).

plemeht,-core of a network is a maximal subset of nodes in the network such enteate each node is connected to at least $k$ others in the subset. $K$-coreness centrality (KC) of a node is $k$ if the node is in $k$-core. Nodes with high $\mathrm{KC}$ in the human PPI network have been found to correspond to "core diseaseome," a subnetwork that is significantly enriched in disease genes and drug targets (Janjić and Pržulj, 2012), as well as to influential "spreaders" of information throughout the network (Kitsak et al., 2010).

Graphlet degree centrality (GDC) measures how many graphlets a node participates in, for all 2-5-node graphlets (Milenković et al., 2011). Intuitively, the more graphlets a node touches, the more central the node is according to GDC. Since it captures the extended network neighborhood of a node, GDC is a highly sensitive measure of network topology. Thus, in a PPI network, proteins with high GDCs represent potential candidates for therapeutic intervention, since targeting such proteins with drugs would have more significant impact on the network structure than targeting proteins that reside in sparse and non-complex network regions (Milenković et al., 2011). Indeed, GDC has been found to capture well disease and pathogeninteracting proteins and drug targets (Milenković et al., 2011).

Betweenness centrality (BETWC) measures the involvement of a node in the shortest paths in the network. Intuitively, nodes that occur in many shortest paths have high centrality according to BETWC. BETWC of node $v$, $C_{\text {betwc }}(v)$, is: $C_{\text {betwc }}(v)=\sum_{s \neq v \neq t \in V} \frac{\sigma_{s t}(v)}{\sigma_{s t}}$, where $V$ is the set of nodes in the network, $\sigma_{s t}$ is the number of shortest paths between nodes $s$ and $t$, and $\sigma_{s t}(v)$ is the number of shortest paths between $s$ and $t$ that go through v. In a PPI network, BETWC of a protein indicates the "likelihood" of the protein to participate in pathways connecting all other proteins (Koschützki and Schreiber, 2008). Removal of a protein that is on critical pathways between many other proteins could cause loss of communication between the proteins. Also, targeting such a node with a drug could cause the drug effects to spread fast to all the nodes (Milenković et al., 2011). This property has been used to identify gene-disease associations by encoding each gene in the network based on the distribution of shortest path lengths to all genes associated with disease (Radivojac et al., 2008). Also, see Kitsak et al. (2010).

Closeness centrality (CLOSEC) measures the "closeness" of a node to all other nodes in the network. Intuitively, nodes with small shortest path distances to all other nodes have high centrality according to CLOSEC. CLOSEC of node $v, C_{\text {closec }}(v)$, is: $C_{\text {closec }}(v)=\frac{1}{\sum_{u \in V} \sigma(u, v)}$, where 
$\sigma(u, v)$ is the shortest path distance between nodes $u$ and $v$. In a PPI network, CLOSEC of a protein indicates the "likelihood" of the protein to reach or be reachable from all other proteins (Scardoni et al., 2009). And it has been a widely accepted assumption that proteins that are closer to each other are more likely to perform the same function (Sharan et al., 2007).

Eccentricity centrality (ECC) is very related to CLOSEC, except that it measures the "closeness" of a node only to the farthest node in the network (Wuchty and Stadler, 2003). Intuitively, nodes with small shortest path distances to the furthest node in the network have high centrality according to ECC. ECC of node $v, C_{\mathrm{ecc}}(v)$, is: $C_{\mathrm{ecc}}(v)=\frac{1}{\max _{u \in V}\{\sigma(u, v)\}}$.

2.3.2 Prediction of aging-related genes For each measure, we compute centrality values for a node in each of the 30 age-specific networks. Then, we calculate Pearson or Spearman correlation between the 30 ages and the node's 30 centrality values (Supplementary Section S1.7). We do this for all genes that are expressed in at least five ages in Lu et al. (2004)'s brain gene expression data (Section 2.1.1). If such a gene is unexpressed at a given age, we assign it a centrality value of zero at that age. Since results are consistent for both correlation measures, here we report results only for Pearson correlation. Results for Spearman correlation are shown in the Supplement.

We quantify the statistical significance of the given correlation value by measuring the probability (i.e., $p$-value) of observing by chance a better value (i.e., the same or higher value when the original value is positive, or the same or lower value when the original value is negative). We do this by randomly reshuffling the 30 node centrality values at the 30 ages and by computing the resulting "random correlation". We repeat this 1,000 times to get 1,000 random correlations. We compute the $p$-value as the percentage of the 1,000 runs in which the random correlation is better than the original one. We predict a gene as aging-related if its $p$-value is below 0.01 .

Since we study multiple node centralities, each of which can predict the given gene as aging-related, we score our predictions so that the more centrality measures support a prediction and the higher the significance of the change of its centrality values with age, the higher the score and the more credible the prediction. For details, see Supplementary Section S1.7.

\subsubsection{Validation of predicted aging-related genes}

Statistical significance of our predictions. To test whether our approach of combining static network data with aging-related expression data into the dynamic network data actually gives meaningful predictions, we study whether the number of aging-related genes that we predict from the actual data is statistically significantly larger than the number of aging-related genes that we predict from "randomized data". By "randomized data", we mean that we randomize the expression data before integrating it with the static network data (Supplementary Section S1.8). Then, we integrate the randomized expression data with the static PPI network, construct randomized age-specific networks just as in Section 2.1.3, and predict agingrelated genes from the randomized networks just as in Section 2.3.2. We repeat the above procedure multiple times, in order to assign a $p$-value to the number of predictions that we make from the actual data (Supplementary Section S1.8).

Overlap between genes of different data sets. We measure the statistical significance of the overlap of genes in one data set and genes in another data set by using the hypergeometric test, which computes probability $p$ (i.e., $p$ value) of observing the same or larger overlap by chance as follows. Let $E$ denote the set of genes that are present in StatNetExpression. $|E|=6,397$. Let $A$ denote the subset of genes in $E$ that are in any one of the two data sets. Let $G$ denote the subset of genes in $E$ that are in the other data set. Let $O$ denote the set of genes that are in the overlap between $A$ and $G$. Then, $p$ is: $p=1-\sum_{i=0}^{|O|-1} \frac{\left(\begin{array}{c}|E| \\ i\end{array}\right)\left(\begin{array}{c}|E|-|A| \\ |G|-i\end{array}\right)}{\left(\begin{array}{c}|E| \\ G \mid\end{array}\right)}$. We use the $p$-value threshold of 0.05 . Gene Ontology (GO) enrichment. We study the enrichment of a data set in biological process GO terms (Ashburner et al., 2000). We use: 1) all
4,913 GO terms that annotate (independent on the evidence code) at least two genes from StatNetExpression and 2) 2,088 GO terms that annotate (with respect to an experimental evidence code only) at least two genes from StatNetExpression. For a GO term $g$, we compute the statistical significance of its enrichment via the above hypergeometric test formula, where now $E$ is the set of genes from StatNetExpression that are annotated by any GO term, $A$ is the gene set in which we are measuring GO term enrichment, $G$ is the subset of genes from $E$ that are annotated by GO term $g$, and $O$ is the set of genes in the overlap between $A$ and $G$. We use the $p$-value threshold of 0.05 .

GO term overlap. We measure the statistical significance of the overlap of GO terms enriched in one data set and GO terms enriched in another data set via the above hypergeometric test formula, where now $E$ is the set of GO terms that annotate at least two genes from StatNetExpression, $A$ is the set of GO terms enriched in any one of the two data sets, $G$ is the set of GO terms enriched in the other data set, and $O$ is the set of GO terms that are in the overlap between $A$ and $G$. We use the $p$-value threshold of 0.05 .

Disease Ontology (DO) enrichment. We study the enrichment of a data set in all 517 DO terms that annotate at least two genes from StatNetExpression (Du et al., 2009) in the same way as when we study GO term enrichments.

DO term overlap. We study the overlap of DO terms from different data sets in the same way as when we study GO term overlaps.

Literature validation. We automatically search for a gene in PubMed (http://www.pubmed.gov) and consider the gene to be validated in the context of aging if its name is mentioned (according to NCBI's E-utilities - http://www.ncbi.nlm.nih.gov/books/NBK25500/) with "age", "aging", or "ageing" in the title or abstract of at least one article. Also, we manually search for a gene by reading relevant PubMed articles more closely.

\section{RESULTS AND DISCUSSIONS}

We study global topologies of the age-specific networks in Section 3.1. We study local topologies of proteins in each network and predict aging-related genes in Sections 3.2.1 and 3.2.2. We validate our predictions in Section 3.2.3.

\subsection{Global network topologies do not change with age}

3.1.1 Global properties of age-specific networks are similar Average clustering coefficients, average diameters, and graphlet frequency distributions (Section 2.2.1) of the age-specific networks do not significantly change with age (Supplementary Fig. S1 and S2).

3.1.2 Networks at different ages belong to the same graph family We compare the fit of the age-specific networks to six network models (Section 2.2.2). The best-fitting model does not change with age (Supplementary Fig. S1 and S2). Note that our primary goal is not to identify the best-fitting model for dynamic PPI networks. Nonetheless, consistent to results for static PPI networks (Pržulj et al., 2010; Kuchaiev et al., 2011; Ratmann et al., 2009), it is gene duplication models that fit the age-specific networks the best.

3.1.3 Overlap of age-specific networks is large The age-specific networks share on average $93 \%$ of the nodes and $90 \%$ of the edges, depending on age, while every pair of the networks shares at least $86 \%$ of the nodes and $79 \%$ of the edges (Supplementary Fig. S3). Hence, the network overlaps are quite large.

\subsection{Local topologies of proteins do change with age}

3.2.1 Prediction of aging-related genes Gene expression data alone revealed only 442 out of thousands of genes as aging-related (Lu et al., 2004). Thus, while global network analysis failed to 


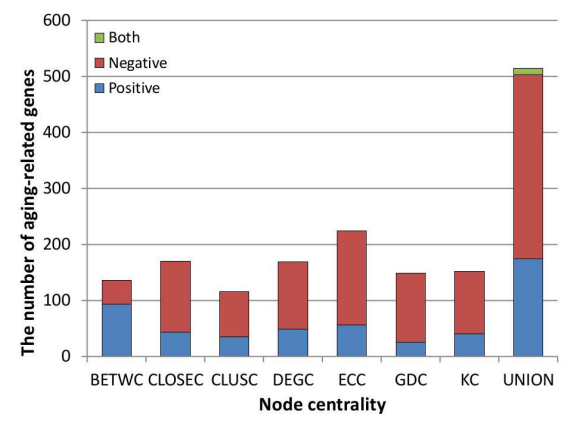

(a)

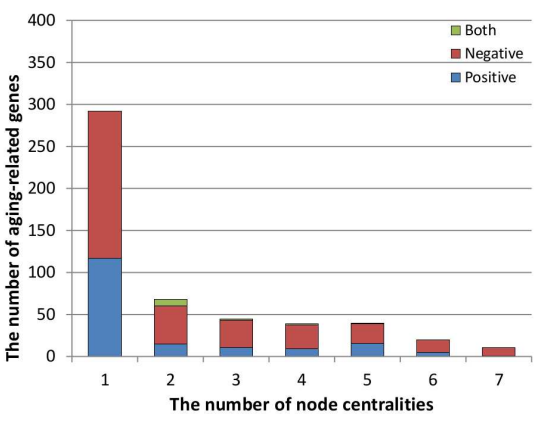

(b)

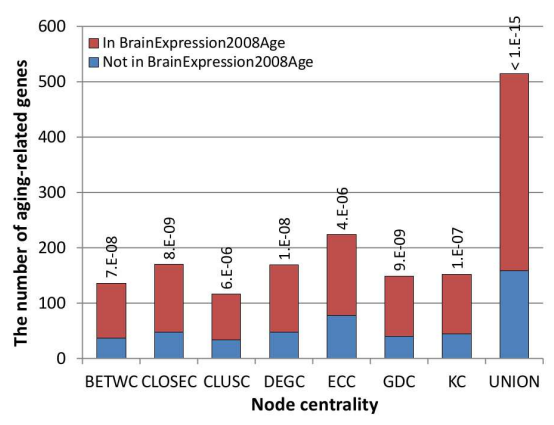

(c)

Fig. 2. The number of our predicted aging-related genes. Panel (a) shows the number of predictions for each of seven node centralities individually (BETWC, CLOSEC, CLUSC, DEGC, ECC, GDC and KC) or by at least one of them (UNION). Panel (b) shows the number of genes predicted by exactly $k$ node centralities $(k=1,2, \ldots, 7)$. In the panels, blue and red bars show the number of genes that are positively and negatively correlated with age, respectively. Green bars denote the number of genes for which one centrality measure identifies the given gene as positively correlated with age, while another measure identifies the same gene as negatively correlated with age. Panel (c) shows the overlaps of our predictions with aging-related genes from BrainExpression2008Age "ground truth" data, for each centrality individually or for all centralities combined. In the panel, blue and red bars show the number of predicted genes that are absent from and present in the "ground truth" data, respectively. The $p$-values for the overlaps are noted at the top of the bars. The results are consistent when we use Spearman correlation to predict aging-related genes instead of Pearson correlation (Section 2.3.2 and Supplementary Fig. S4). Also, the results are consistent when we use BioGRID data as the static PPI network instead of HPRD data (Section 2.1.3 and Supplementary Fig. S5).

uncover any aging-related information, it could be that the dynamic network data encodes aging-related information only locally and around only a subset of nodes. So, we use node centrality measures (Section 2.3.1) to quantify local positions of nodes in the agespecific networks and find nodes whose centralities correlate well with age, as such proteins could be key players in aging.

We predict a gene as aging-related if its centrality values are statistically significantly correlated with age (Section 2.3.2) for at least one centrality measure. This results in $515(8.1 \%)$ predictions out of all 6,397 genes. Fig. 2 (a) shows the number of aging-related predictions for each centrality individually and all centralities combined. No centrality predicts drastically more genes than others.

A gene's centrality can be positively correlated with age (the gene becomes more network-central with age) or it can be negatively correlated with age (the gene becomes less central with age). The majority of our predictions are negatively correlated with age (Fig. 2). This finding is encouraging, since it has already been argued that aging is associated with failure of "hubs" - highly interconnected and thus network-central proteins (Soltow et al., 2010).

\subsubsection{Relationships and potential redundancies of different node} centralities We predict a gene as aging-related if its centrality values correlate well with age with respect to at least one centrality. So, we study whether any genes are predicted by more than one or even all of the centralities. We find that almost half $(43 \%)$ of the 515 aging-related predictions are supported by multiple centralities, while the remaining predictions are supported by a single centrality (Fig. 2 (b)). As expected, the number of predictions decreases as the number of centralities supporting the predictions increases.

We study the redundancy of the different centralities by computing, for each pair of centralities, the correlation between their centrality values over all nodes in the given network, and by averaging correlations over the 30 age-specific networks. We observe high correlations between some measures, such as DEGC, KC, and GDC, or CLOSEC and ECC (Fig. 3 (a) and Supplementary Section S2.1). Thus, some centralities appear to be redundant to others.
However, when we study pairwise overlaps of aging-related predictions produced by the different centralities, the overlaps are not very large for any pair of centralities (Fig. 3 (b)). This result, together with the result from Fig. 2 (b), which shows that the majority $(\sim 57 \%)$ of the predictions are identified by a single centrality, suggests that the centralities are not redundant to each other. When we focus on our aging-related genes predicted by exactly one centrality measure, in most cases, these predictions are not even marginally significant with respect to other centrality measures (Fig. 3(c) and Supplementary Fig. S6), indicating again that the different centralities are in general not redundant to each other. Thus, we keep all 515 predictions, independent on the number of centralities supporting the given prediction. Henceforth, we denote this set of 515 aging-related genes predicted via dynamic network analysis as DyNetAge. We denote the complement of this set, i.e., the set of genes that are present in StatNetExpression but not in DyNetAge, as DyNetComplement. Clearly, DyNetComplement is the set of genes whose centralities do not significantly correlate with age, and as such, we do not predict them as aging-related.

Next, we study the effect of the number of centralities supporting a prediction on the quality of the prediction. It is not necessarily the case that predictions supported by many centralities are more enriched in "ground truth" aging-related genes (see below) compared to predictions supported by only few centralities (Supplementary Fig. S7). Nonetheless, it is the case that the more centralities support a prediction, the more significant the correlation of its centrality values with age (as indicated by lower $p$-values in Fig. 3(d)). Hence, to account for the number of centralities supporting a prediction, we rank the prediction so that the more centralities support it and the more significant the change of its centrality values with age, the more credible the prediction (Section 2.3.2). (Supplementary Tables S1 and S2 contain the ranked lists of all predictions.) We validate our scoring scheme by demonstrating that our $10 \%$ highest-scoring predictions are statistically significantly enriched in genes that are present in more 
than one "ground truth" data set ( $p$-value of 0.003 ), whereas this is not the case for the lower-scoring predictions.

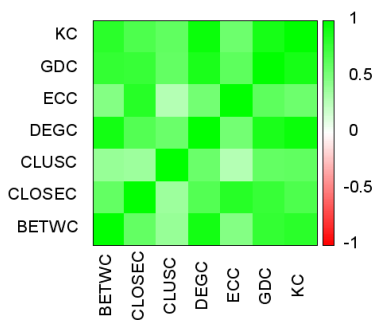

(a)

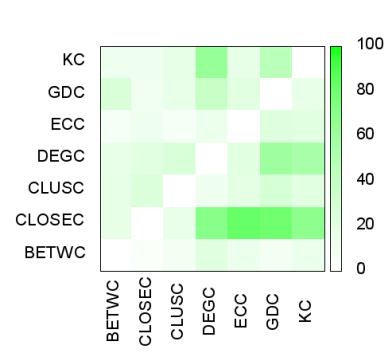

(c)

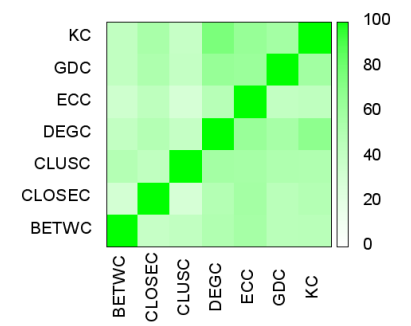

(b)

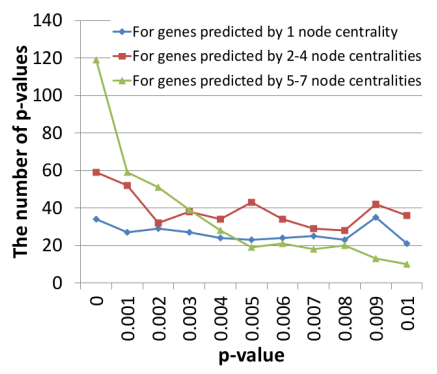

(d)
Fig. 3. Relationships between different centralities (BETWC, CLOSEC, CLUSC, DEGC, ECC, GDC and KC): (a) Spearman correlation between each pair of centralities averaged over the 30 age-specific networks; (b) pairwise overlap of aging-related genes predicted by the different centralities; (c) percentage of genes predicted as aging-related ( $p$-value $\leq 0.01$ ) by exactly one centrality (listed in a given row), which have a "marginal" $p$-value between 0.01 and 0.05 with respect to one of the six remaining centralities (listed in a given column), and (d) distributions of $p$ values of our predicted aging-related genes. Panel (c) can be interpreted as follows. For example, many of the predictions identified only by CLOSEC (the second last row) are marginally significant with respect to DEGC, ECC, GDC, and KC (intensive color), while almost none of the predictions identified only by BETWC (the last row) are marginally significant with respect to any other centrality (light color). Hence, the predictions made by CLOSEC only may be more credible than the predictions made by BETWC only, as the former are marginally supported by additional centralities, but the latter are not.

\subsubsection{Validation of predicted aging-related genes}

Dynamic network analysis gives meaningful and statistically significant aging-related predictions. The number of agingrelated genes in DyNetAge is statistically significantly larger than the number of aging-related genes that we can predict from the "randomized" data $\left(Z\right.$-score $=7.42, p$-value $<10^{-10}$; Section 2.3.3).

Overlap of our predictions with "ground truth" aging-related genes is statistically significant. Human aging is hard to study experimentally due to long life span and ethical constraints. Instead, human aging-related "ground truth" knowledge has been predicted more or less computationally, by studying gene expression data or by transferring aging-related knowledge from model species to human via sequence comparison. (We intentionally use quotes, as we are not dealing with true, experimentally obtained ground truth data.) Similarly, here, we aim to computationally predict new "ground truth" aging-related data from an additional data type
- PPIs. (But by no means do we claim to identify all agingrelated genes.) Hence, since "ground truth" data sets are predicted computationally, they could be noisy. Also, different "ground truth" sets could be biased towards different data types from which the predictions have been made, be it expression, sequence, or PPI data. Since different data types could be capturing different functional slices of the cell (Memišević et al., 2010; Przytycka and Kim, 2010), it might not be alarming if the intersections between the different "ground truth" sets are not very large. However, since all the sets are aiming to capture the same biological phenomenon (aging), some overlap would be encouraging. Ideally, we would like to see a statistically significant overlap. However, the existence of some overlap would be encouraging even if the overlap was not significant, since: (1) the overlap could be low due to the noisiness of each of the "ground truth" sets, and (2) statistically non-significant results may be biologically important, whereas statistically significant results may not be (Motulsky, 1995; Milenković et al., 2010a; Ho et al., 2010).

We measure the overlap of DyNetAge with five "ground truth" data sets: BrainExpression2004Age, BrainExpression2008Age, ADExpressionAge, HGPSExpressionAge, and SequenceAge (Section 2.1.4). Of the five, BrainExpression2004Age and BrainExpression2008Age are the most likely to be similar to DyNetAge (as all three are based on brain-related data, brainrelated gene expression data, and brain aging-related gene expression data; Supplementary Section S2.2), followed by ADExpressionAge (as our predictions as well as this data are both based on brain-related data and brain-related gene expression data), followed by HGPSExpressionAge (as our predictions as well as this data are both based on gene expression data), followed by SequenceAge (as our predictions and this data both capture aging-related information but from different data types) (Section 2.1.4). Therefore, a high overlap of DyNetAge with BrainExpression2004Age or BrainExpression2008Age would validate our method. A high overlap with ADExpressionAge would suggest that our method could capture not only brain aging-related genes but also brain aging-related disease genes. A high overlap with HGPSExpressionAge would suggest that our method could capture genes related to a tissue different from the brain tissue. A high overlap with SequenceAge would suggest that our method could capture genes identified from a different data type, namely sequence data.

As hypothesized, DyNetAge overlaps the most (as indicated by the lowest $p$-values) with BrainExpression2004Age and BrainExpression2008Age, followed by ADExpressionAge, HGPSExpressionAge, and SequenceAge, respectively (Supplementary Fig. S8 and Supplementary Table S3). The overlap is statistically significant for BrainExpression2004Age, BrainExpression2008Age, and ADExpressionAge, marginally significant for HGPSExpressionAge, and non-significant for SequenceAge (Table 1). The (marginally) significant overlap between DyNetAge and four out of the five "ground truth" data sets is encouraging. Importantly, even though most of the overlaps are statistically significant, $87 \%$, $31 \%, 74 \%, 87 \%$, and $96 \%$ of our DyNetAge predictions are not in BrainExpression2004Age, BrainExpression2008Age, ADExpressionAge, HGPSExpressionAge, and SequenceAge, respectively, and 19\% of our predictions are not in any of the five data sets (Supplementary Table S4). This confirms that data 
integration can reveal additional biological knowledge compared to studying individual data types.

Some overlap between DyNetAge and SequenceAge is also encouraging, even though the overlap is non-significant (as argued above). Further, the overlap is stronger between DyNetAge and the other four "ground truth" data sets (BrainExpression2004Age, BrainExpression2008Age, ADExpressionAge, and HGPSExpressionA than between SequenceAge and these four "ground truth" data sets. In particular, whereas DyNetAge overlaps significantly with three of the four data sets and marginally significantly with respect to the fourth data set (see above), SequenceAge overlaps significantly with just one of the four data sets (ADExpressionAge), almost marginally significantly with respect to two of the four data sets (HGPSExpressionAge and BrainExpression2004Age), and non-significantly with respect to the remaining data set (BrainExpression2008Age) (Supplementary Table S3). Therefore, our DyNetAge appears to be more relevant than SequenceAge with respect to the other "ground truth" data sets. The non-significant overlaps could be due to potential complementarity of the different types of biological data, noisiness of the "ground truth" data, or some of the "ground truth" data sets being biased towards brain-related genes.

Recall that the complement of each "ground truth" data set (including DyNetAge) is the set of genes not predicted as agingrelated by the given study (Section 2.1.5). Hence, it would be encouraging to see: 1) low (non-significant) overlaps between DyNetAge and complements of the "ground truth" data sets, 2) low (non-significant) overlaps between DyNetComplement and "ground truth" sets, and 3) high (significant) overlaps between DyNetComplement and complements of "ground truth" sets. Indeed, this is what we typically observe in all three cases (Supplementary Table S3).

Table 1. Overlap and its statistical significance (i.e. $p$-value) between aging-related genes (ARG), GO terms (GO), and DO terms (DO) in DyNetAge and those in the five "ground truth" aging-related data sets (BrainExpression2004Age (BE4A), BrainExpression2008Age (BE8A), ADExpressionAge (ADEA), HGPSExpressionAge (HEA), and SequenceAge (SA)).

\begin{tabular}{|c|c|c|c|c|c|c|}
\hline & & BE4A & BE8A & ADEA & HEA & SA \\
\hline \multirow{2}{*}{ ARG } & Overlap & $20 \%$ & $69 \%$ & $26 \%$ & $13 \%$ & $9 \%$ \\
& $p$-value & $5.5 \mathrm{E}-13$ & $<1 \mathrm{E}-15$ & $4.3 \mathrm{E}-7$ & 0.06 & 0.39 \\
\hline GO & Overlap & $20 \%$ & $21 \%$ & $12 \%$ & $6 \%$ & $17 \%$ \\
& $p$-value & $1.2 \mathrm{E}-10$ & $2.5 \mathrm{E}-14$ & $2.8 \mathrm{E}-6$ & 0.28 & 0.33 \\
\hline \multirow{2}{*}{ DO } & Overlap & $25 \%$ & $25 \%$ & $0 \%$ & $0 \%$ & $25 \%$ \\
& $p$-value & 0.03 & $7.2 \mathrm{E}-3$ & N/A & N/A & 0.70 \\
\hline
\end{tabular}

GO enrichment. When analyzing all gene-GO term associations, 146 GO terms are significantly enriched in DyNetAge ( $p$-values between 0.047 and $\left.1.5 \times 10^{-5}\right)$, while only $14 \mathrm{GO}$ terms are enriched in DyNetComplement ( $p$-values between 0.043 and $5.1 \times$ $10^{-5}$ ) (Section 2.3.3). Importantly, there is no overlap between the GO terms from DyNetAge and those from DyNetComplement. Hence, our aging-related predictions group by functions that are different than functions of genes that we do not predict as agingrelated.

When we focus on aging-related GO terms only, it is encouraging that DyNetAge contains genes annotated with aging, cell aging, and cellular senescence GO terms, as well as that DyNetAge's performance is typically comparable to that of the "ground truth" data, especially when using only gene-GO term associations obtained by experimental evidence codes (Supplementary Section $\mathrm{S} 2.3$ ).

GO overlap. Our predictions are further validated by: 1) high overlaps between GO terms from DyNetAge and GO terms prom the "ground truth" data sets, 2) low overlaps between GO terms from DyNetAge and GO terms from complements of the "ground truth" sets, 3) low overlaps between GO terms from DyNetComplement and GO terms from the "ground truth" sets, and 4) high overlaps between GO terms from DyNetComplement and GO terms from complements of the "ground truth" sets (Table 1 and Supplementary Table S5). For example, when considering gene-GO term associations of any evidence code, GO terms from DyNetAge significantly overlap with GO terms from three of the five "ground truth" data sets (BrainExpression2004Age, BrainExpression2008Age, and ADExpressionAge), and some (though non-significant) overlap with GO terms from HGPSExpressionAge and SequenceAge is also encouraging (Table 1). Equivalent results when considering gene-GO term associations of experimental evidence codes only are shown in Supplementary Table S6. Importantly, in this case, SequenceAge fails to significantly overlap with any other "ground truth" set, whereas DyNetAge still significantly overlaps with BrainExpression2008Age. For details, see Supplementary Section S2.4.

DO enrichment. Eight diseases are significantly enriched in DyNetAge $\left(p\right.$-values between 0.041 and $\left.6 \times 10^{-3}\right)$, while 14 diseases are enriched in DyNetComplement ( $p$-values between 0.049 and $2.9 \times 10^{-3}$ ) (Section 2.3.3). Importantly, there is no overlap between diseases from DyNetAge and those from DyNetComplement.

The eight diseases in DyNetAge are: brain disease, brain tumor, bipolar disorder, connective tissue disease, renal tubular acidosis, leukodystrophy, neuroblastoma, and demyelinating disease. Brain disease and renal tubular acidosis are enriched in SequenceAge as well, brain disease and bipolar disorder are enriched in BrainExpression2004Age as well, and brain tumor and neuroblastoma are enriched in BrainExpression2008Age as well. All of these overlaps are encouraging. (We quantify the significance of the overlaps in the following section.) In particular, renal tubular acidosis, whose aging-related evidence is supported by SequenceAge, is kidney-related, whereas DyNetAge has been predicted from the brain-related data. Capturing this non-brainrelated disease in DyNetAge is encouraging, especially because BrainExpression2004Age and BrainExpression2008Age fail to do so. Further aging-related evidence for these five diseases can be found in the following references denoted by their PubMed IDs (PMIDs): 11256685, 8040891, 21197651, and 21031036. Supplementary Table S7 maps PMIDs to full paper references. Importantly, even three diseases from DyNetAge that are missed by all "ground truth" sets can all be linked to aging in the literature as well (Supplementary Section S2.5).

DO overlap. We further validate our predictions by demonstrating: 1) high overlaps between $D O$ terms from DyNetAge and DO terms from the "ground truth" data sets, 2) low overlaps between DO terms from DyNetAge and DO terms from complements of the "ground truth" sets, 3) low overlaps between DO terms from 
DyNetComplement and DO terms from the "groung truth" sets, and 4) high overlaps between DO terms from DyNetComplement and DO terms from complements of the "ground truth" sets (Supplementary Table S8). For example, DO terms from DyNetAge statistically significantly overlap with DO terms from two of the five "ground truth" data sets (BrainExpression2004Age and BrainExpression2008Age) (Table 1). It is not alarming that DyNetAge does not significantly overlap with ADExpressionAge, HGPSExpressionAge, or SequenceAge, since none of the three overlaps significantly with more than one of the five "ground truth" data sets (Supplementary Table S8). Hence, DyNetAge is better supported by the "ground truth" data than any of these three data sets. For details, see Supplementary Sections S2.6 and S2.7.

Literature validation. Automatic literature validation (Section 2.3.3) is prone to errors: we "validate" in this manner equal portion of both the "ground truth" aging-related sets and their complements. Therefore, we aim to validate our predictions manually (Section 2.3.3). Since manual validation is laborious, we focus on our $10 \%$ highest-scoring predictions (Section 2.3.2). Of these, we study predictions that are absent from all "ground truth" sets, namely DVL1, ACACA, HOMER3, GJB1, FKBP8, and H1F0. We successfully validate all of these genes. DVL1 has been linked to Alzheimer's disease (PMID: 11803455), which has been linked to aging itself (PMID: 21197651, 21031036). Expression level of ACACA in rat changes with age (PMID: 11044254), and so its human ortholog is an aging-related candidate. HOMER$1 \mathrm{~A}$, a member of the same family as HOMER3, affects the level of cognitive performance during aging (PMID: 23054826). The expression of GJB1 is down-regulated with aging (PMID: 22337502). Drug targeting of FKBP38 might successfully intervene with FKBP38-dependent processes such as programmed cell death in cancer or neurodegenerative diseases (PMID: 21514222), which have been linked to aging (PMID: 21197651, 21031036). H1F0 has been linked to age-related macular degeneration (PMID: 16518403), and its rat ortholog causes aging-related alterations in liver (PMID: 8114518).

\section{CONCLUSION}

Together, our results confirm that dynamic PPI network analysis via integration of static PPI network data with aging-related gene expression data can reveal meaningful key players in aging.

Funding: NSF EAGER CCF-1243295 grant.

\section{REFERENCES}

Aragues, R., Sander, C. and Oliva, B. (2008) Predicting cancer involvement of genes from heterogeneous data. BMC Bioinformatics, 9, 172.

Ashburner, M. et al. (2000) Gene ontology: tool for the unification of biology. Nature Genetics, 25, 25-29.

Barabási, A. L. and Oltvai, Z. (2004) Network biology: Understanding the cell's functional organization. Nature Reviews, 5, 101-113.

Berchtold, N. C. et al. (2008) Gene expression changes in the course of normal brain aging are sexually dimorphic. PNAS, 105, 15605-10.

Breitkreutz, B. J. et al. (2008) The BioGRID Interaction Database: 2008 update. Nucleic Acids Research, 36, D637-D640.

de Magalhães, J. (2009) Aging research in the post-genome era: New technologies for an old problem. In Foyer, C., Faragher, R. and Thornalley, P. (eds.), Redox Metabolism and Longevity Relationships in Animals and Plants, pp. 99-115. Taylor and Francis, New York.

de Magalhães, J. et al. (2009) The Human Ageing Genomic Resources: online databases and tools for biogerontologists. Aging Cell, 8, 65-72.

Du, P. et al. (2009) From disease ontology to disease-ontology lite: statistical methods to adapt a general-purpose ontology for the test of gene-ontology associations.
Bioinformatics, 25, i63-68.

Dyer, M., Murali, T. and Sobral, B. (2008) The landscape of human proteins interacting with viruses and other pathogens. PLoS Pathog, 4, e32+.

Ferrarini, L., Bertelli, L., Feala, J., McCulloch, A. D. and Paternostro, G. (2005) A more efficient search strategy for aging genes based on connectivity. Bioinformatics, 21 338-348.

Fortney, K., Kotlyar, M. and Jurisica, I. (2010) Inferring the functions of longevity genes with modular subnetwork biomarkers of Caenorhabditis elegans aging. Genome Biology, 11, R13+

Goh, K. et al. (2007) The human disease network. PNAS, 104, 8685-8690.

Ho, H. et al. (2010) Protein interaction network uncovers melanogenesis regulatory network components within functional genomics datasets. BMC Systems Biology, 4.

Janjić, V. and Pržulj, N. (2012) The core diseasome. Molecular bioSystems, 8, 2614-25.

Jeong, H., Mason, S. P., Barabási, A. L. and Oltvai, Z. N. (2001) Lethality and centrality in protein networks. Nature, 411, 41-2.

Jonsson, P. F. and Bates, P. A. (2006) Lobal topological features of cancer proteins in the human interactome. Bioinformatics, 22, 2291-2297.

Kitsak, M. et al. (2010) Identification of influential spreaders in complex networks. Nature Physics, 6, 888-893.

Koschützki, D. and Schreiber, F. (2008) Centrality analysis methods for biological networks and their application to gene regulatory networks. Gene Regulation and Systems Biology, 2, 193-201

Kriete, A., Lechner, M., Clearfield, D. and Bohmann, D. (2011) Computational systems biology of aging. Wiley Interdiscip Rev Syst Biol Med, 3, 414-28.

Kuchaiev, O., Stevanović, A., Hayes, W. and Pržulj, N. (2011) GraphCrunch 2: Software tool for network modeling, alignment and clustering. BMC Bioinformatics, 12

Liu, G.-H. et al. (2011) Recapitulation of premature ageing with ipscs from hutchinsongilford progeria syndrome. Nature, 472, 221-5.

Lu, T. et al. (2004) Gene regulation and DNA damage in the ageing human brain Nature, 429, 883-891.

Memisević, V., Milenković, T. and Pržulj, N. (2010) An integrative approach to modeling biological networks. Journal of Integrative Bioinformatics, 7, 120.

Memišević, V., Milenković, T. and Pržulj, N. (2010) Complementarity of network and sequence information in homologous proteins. Journal of Integrative Bioinformatics, 7, 135.

Milenković, T., Filippis, I., Lappe, M. and Pržulj, N. (2009) Optimized null model for protein structure networks. PLoS ONE, 4, e5967.

Milenković, T., Lai, J. and Pržulj, N. (2008) GraphCrunch: a tool for large network analyses. BMC Bioinformatics, 9.

Milenković, T., Memisević, V., Ganesan, A. K. and Pržulj, N. (2010a) Systems-level cancer gene identification from protein interaction network topology applied to melanogenesis-related interaction networks. Journal of the Royal Society Interface, 7, 423-437.

Milenković, T., Memišević, V., Bonato, A. and Pržulj, N. (2011) Dominating biological networks. PLOS ONE, 6, e23016.

Milenković, T., Ng, W., Hayes, W. and Pržulj, N. (2010b) Optimal network alignment with graphlet degree vectors. Cancer Informatics, 9, 121-137.

Milenković, T. and Pržulj, N. (2008) Uncovering biological network function via graphlet degree signatures. Cancer Informatics, 6, 257-273.

Motulsky, H. (1995) Intuitive Biostatistics. Oxford University Press, 1 edition.

Pastor-Satorras, R., Smith, E. and Sole, R. V. (2003) Evolving protein interaction networks through gene duplication. Journal of Theoretical Biology, 222, 199-210.

Peri, S. et al. (2004) Human protein reference database as a discovery resource for proteomics. Nucleic Acids Res, 32 Database issue, D497-501. 1362-4962Journal Article.

Promislow, D. (2004) Protein networks, pleiotropy and the evolution of senescence. Proceedings of the Royal Society B: Biological Sciences, 1545, 1225-1234.

Pržulj, N. (2007) Biological network comparison using graphlet degree distribution. Bioinformatics, 23, e177-e183.

Pržulj, N. (2011) Protein-protein interactions: Making sense of networks via graphtheoretic modeling. Bioessays, 33, 115-123.

Pržulj, N., Kuchaiev, O., Stevanović, A. and Hayes, W. (2010) Geometric evolutionary dynamics of protein interaction networks. Pacific Symposium on Biocomputing, pp. 178-89.

Przytycka, T. and Kim, Y. (2010) Network integration meets network dynamics. BMC Biology, 8, 48+.

Radivojac, P. et al. (2008) An integrated approach to inferring gene-disease associations in humans. Proteins, 72, 1030-1037. 
Ratmann, O., Wiuf, C. and Pinney, J. W. (2009) From evidence to inference: probing the evolution of protein interaction networks. HFSP Journal, 3, 290-306.

Reja, R. et al. (2009) MitoInteractome: mitochondrial protein interactome database, and its application in 'aging network' analysis. BMC genomics, 10 Suppl 3, S20+.

Scardoni, G., Petterlini, M. and Laudanna, C. (2009) Analyzing biological network parameters with centiscape. Bioinformatics, 25, 2857-2859.

Sharan, R. and Ideker, T. (2008) Protein networks in disease. Genome Research, 18 $644-652$.

Sharan, R., Ulitsky, I. and Shamir, R. (2007) Network-based prediction of protein function. Molecular Systems Biology, 3, 1-13.

Sốti, C. and Csermely, P. (2007) Aging cellular networks: Chaperones as major participants. Experimental Gerontology, 42, 113-119.

Simpson, J. E. et al. (2011) Microarray analysis of the astrocyte transcriptome in the aging brain: relationship to alzheimer's pathology and apoe genotype. Neurobiology of Aging, 32, 1795-807.

Soltow, Q., Jones, D. and Promislow, D. (2010) A Network Perspective on Metabolism and Aging. Integrative and comparative biology, 50, 844-854.

Vanunu, O., Magger, O., Ruppin, E., Shlomi, T. and Sharan, R. (2010) Associating genes and protein complexes with disease via network propagation. PLoS Computational Biology, 6, e1000641.

Wieser, D., Papatheodorou, I., Ziehm, M. and Thornton, J. (2011) Computational biology for ageing. Philosophical Transactions of the Royal Society B: Biological Sciences, 366, 51-63.

Wuchty, S. and Stadler, P. F. (2003) Centers of complex networks. Journal of Theoretical Biology, 223, 45-53. 